\title{
Effect of Reactor Neutrinos on Beta-Decay
}

\author{
Boris V. Vasiliev \\ Dubna, Russia \\ Email: bv.vasiliev@yandex.com
}

How to cite this paper: Vasiliev, B.V. (2020) Effect of Reactor Neutrinos on Beta-Decay. Journal of Modern Physics, 11, 91-96.

https://doi.org/10.4236/jmp.2020.111005

Received: November 23, 2019

Accepted: January 7, 2020

Published: January 10, 2020

Copyright $\odot 2020$ by author(s) and Scientific Research Publishing Inc. This work is licensed under the Creative Commons Attribution International License (CC BY 4.0).

http://creativecommons.org/licenses/by/4.0/

\begin{abstract}
The pulsed nuclear reactor was used to measure the effect of neutrinos on the beta-decay of ${ }^{90} \mathrm{Sr} /{ }^{90} \mathrm{Y}$ nuclei. This measurement shows that some increase in the decay rate occurs in a few tens of milliseconds after reactor flashes.
\end{abstract}

\section{Keywords}

Neutrino, Pulse Nuclear Reactor, Short-Lived Isotope, Beta-Decay

\section{Introduction}

\subsection{Beta-Decay}

The state of radioactive nuclei is energetically unstable. At decay, they pass from a certain excited state to a state with lower energy.

The question arises: what is the reason that at the some moment there is a decay of a particular nucleus? And in general, is such a deterministic approach to this problem legitimate?

The assumption that the cause of the decay of radioactive nuclei may be their interaction with the neutrino flux was repeatedly expressed earlier [1] [2] [3].

It can be assumed that in order to cause a radioactive decay occurring with the energy releasing, a quasi-elastic collision of neutrino with a nucleus is enough.

The electromagnetic model of neutron [4] allows to calculate all its main parameters-mass, spin, magnetic moment, decay energy. Only the time of its life defies calculation.

According to this model, proton and electron forming the neutron are bound by electromagnetic forces. These forces do not carry any mechanism of degrada- 
tion of the bound state. There is no internal cause that could cause the loss of stability of neutron and its decay.

Therefore, we can assume that $\beta$-decay is a forced phenomenon that occurs under the external influence of the neutrino flux.

This assumption can be verified if the correlation between the neutron lifetime or the rate of the nuclei $\beta$-decay and the intensity of the neutrino flux incident on them can be studied.

\subsection{The Neutron Time of Life}

Careful measurements of the neutron lifetime were carried out repeatedly. The results of these measurements, carried out at different reactors, differ markedly.

According to measurements made in the past century at relatively small reactors, the average neutron lifetime is $885.7 \pm 0.8 \mathrm{~s}$ [5].

In 2005 , these measurements were repeated on the powerful research reactor in Grenoble.

These measurements showed that the neutron lifetime is equal to $878.5 \pm 0.7$ $\pm 0.3 \mathrm{sec}[5]$.

It can be assumed that the discrepancy is caused by a more powerful neutrino flux of the Grenoble reactor.

\subsection{The Effect of Solar Neutrinos on $\beta$-Decay}

Extremely interesting results were published by E. Fischbach, J. H. Jenkins et al. [1].

According to experiments conducted earlier at different times and in different countries of the world, the nuclear decay rate under terrestrial conditions is variable. It depends on the Earth-Sun distance.

Summarizing these measurement data, authors [1] expressed a hypothesis about the possible influence of the solar neutrino flux on the nuclear decay rate.

Accordingly to measurement data [6], the sunny neutrino flux is

$$
\Phi_{\odot} \approx 6 \times 10^{10} \mathrm{v} / \mathrm{cm}^{2} \cdot \mathrm{s} .
$$

Proceeding from the Fischbach-Jenkins assumption, then the rate of measured in our laboratories beta-decay should change throughout the year due to the movement of the Earth in the elliptical orbit around the Sun. Since the difference between the distances to the Sun at perihelion and aphelion is about 3.5\%, then the corresponding solid angle of the earth laboratory changes by approximately $7 \%$ at the same time. If the $\beta$-decay of nuclei would be caused only by the solar neutrino flux, then we can expect that the rate of $\beta$-decay in the terrestrial laboratory must be modulated to a depth of $7 \%$ with a period equal to year.

It is remarkable that the experiments basically confirmed the Fischbach-Jenkins theory. The decay rate of a number of nuclei really turned out to be sinusoidally modulated with a period equal to year.

The only difference was that the modulation depth was about 10 times smaller. 
This can be explained if we assume that the solar neutrino flux is only $1 / 10$ of the total neutrino flux, which affects the decay of nuclei in our laboratories, and the total neutrino flux from all cosmic sources

$$
\Phi_{\text {cosmic }} \approx 10^{12} v / \mathrm{cm}^{2} \cdot \mathrm{s} \text {. }
$$

\section{Measurement of the Effect of Reactor Neutrinos on $\beta$-Decay}

\subsection{The Flux of Reactor Neutrinos}

Neutrinos emitted by nuclear reactors usually have fluxes that are much smaller than cosmic neutrinos, but the energy of reactor neutrinos is higher [7].

In our experiment, the pulse reactor IBR-2 (Dubna) was used as the neutrino source. Its average power was equal to

$$
W_{\text {IBR-2 }} \approx 1.6 \mathrm{MW} \approx 10^{19} \mathrm{MeV} / \mathrm{sec} .
$$

Short explosions of its activity follow one after the other every

$$
\tau_{r}=0.2 \mathrm{~s} .
$$

The duration of the flash was equal to

$$
\tau_{f} \approx 300 \mathrm{mks} \text {. }
$$

As fission of one nucleus into nuclear fuel releases energy [7] [8]

$$
E_{f} \approx 200 \mathrm{MeV}
$$

the reactor produces

$$
N_{f} \approx 5 \times 10^{16} \text { fission } / \mathrm{s}
$$

acts of fission per second.

The mechanism leads to the generation of neutrinos in the fission reaction, due to the fact that $\beta$-active nuclei born as fragments of the fuel fission.

However, basically these fragments in our case can be considered as long-lived because their half-life is longer than the period between our reactor flashes $\tau_{r}$. Neutrinos emitted during the decay of these $\beta$-active nuclei are not "tied" to a specific reactor flash.

They created in our experiment a small increase in the background.

There are few short-lived isotopes that have a half-life of $T_{1 / 2}<\tau_{r} \quad$ [9]. These are, for example, the nuclei ${ }^{12} \mathrm{~B}\left(T_{1 / 2} \approx 20 \mathrm{~ms}\right)$.

If we assume that one act of plutonium fission produces one short-lived isotope, emitting one neutrino during its decay, then the flux of these neutrinos $\Phi_{\text {react }}$ in the experimental hall of the reactor at a distance of about 20 meters from its core will be only a small part of the flux of cosmic neutrinos (Equation (2)):

$$
\frac{\Phi_{\text {react }}}{\Phi_{\text {cosm }}} \approx 1.5 \times 10^{-3} .
$$

However, the effect of this flux can be registered, since the impact of the pulse reactor can be accumulated if the addition of the measurement data is carried 
out synchronously with the reactor flashes.

The discriminator that sets the threshold for recording photomultiplier pulses played an important role in the setup we used (Figure 1). The nuclei of strontium-90 at beta-decay starts a chain of transformations:

$$
{ }^{90} \mathrm{Sr} \rightarrow{ }^{90} \mathrm{Y} \rightarrow{ }^{90} \mathrm{Zr}
$$

The energy distribution of beta electrons in this chain is shown in Figure 2.

The presence of a significant difference in the energy of beta-electrons formed during the decay of ${ }^{90} \mathrm{Sr}$ and ${ }^{90} \mathrm{Y}$ atoms gives rise to the assumption that the effect of reactor neutrinos on these decays may also be different.

In order to register this difference, the influence of reactor neutrinos on beta-decay was measured at different levels of beta-electron pulse energy discrimination (Figure 3 ). The exponential component of the complete set of measured data was obtained by computer calculation.

It should be noted that curve 5 in Figure 3 when constructed on a large scale is also an exponent with a small pre-exponential multiplier.

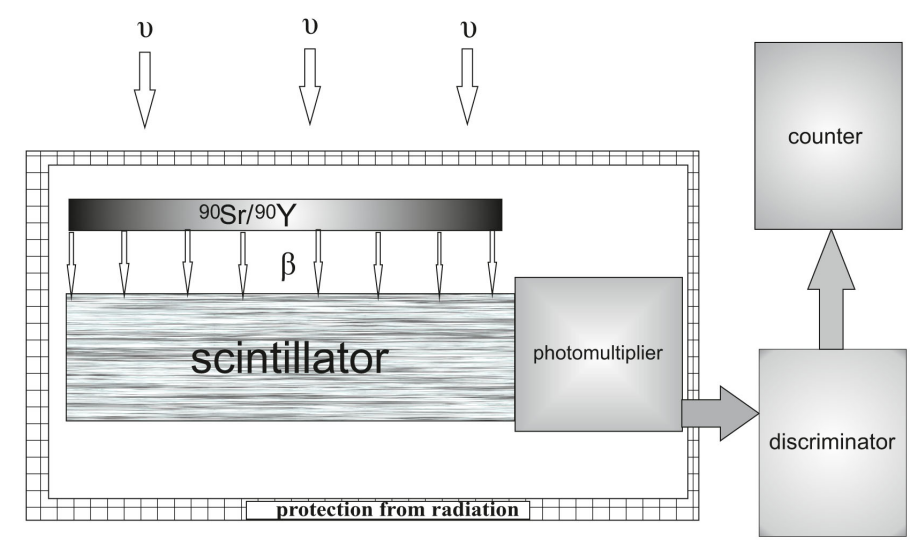

Figure 1. The scheme of measuring equipment.

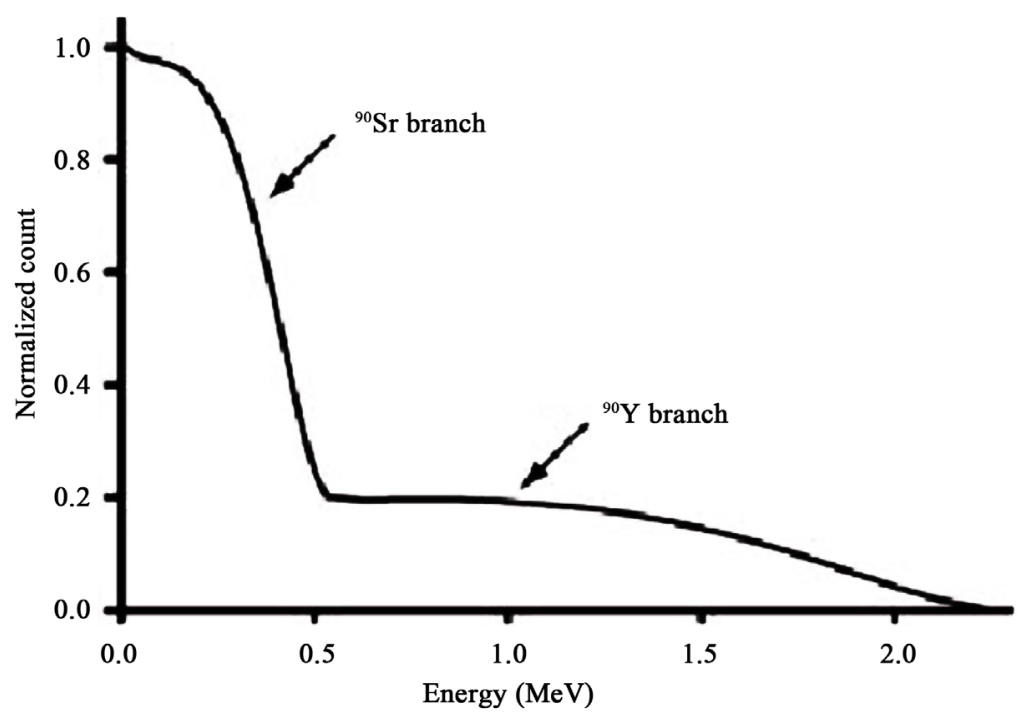

Figure 2. The energy distribution of beta-electrons generated by ${ }^{90} \mathrm{Sr} /{ }^{90} \mathrm{Y}$ nuclei. 


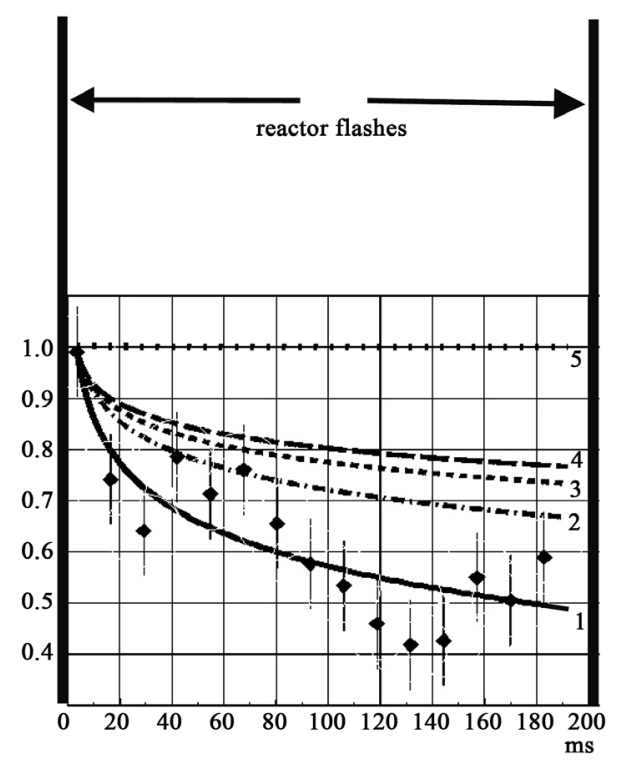

Figure 3. The result of the accumulation of registered data for beta-electrons emitted by the ${ }^{90} \mathrm{Sr} /{ }^{90} \mathrm{Y}$-source in the time interval between reactor flashes. Accumulation was carried out in various cases from 1 day to almost 6 days. The curves in this figure are exponential components extracted by computer from a complete set of measured data. The full sets of these data were obtained at different levels of discrimination $\mathrm{D}$. The curve $1: \mathrm{D}=0.2-0.3$ $\mathrm{MeV}$, curves 2, 3 and 4: $\mathrm{D}=0.4-0.5 \mathrm{MeV}$, the curve 5: $\mathrm{D}=1.5-2 \mathrm{MeV}$.

\subsection{Experimental Equipment and Measurement Results}

In our experiment, the search was made for a correlation between the rate of $\beta$-decay of ${ }^{90} \mathrm{Sr} /{ }^{90} \mathrm{Y}$ isotopes and activity pulses of nuclear reactor.

For this, the $\beta$-electrons emitted by ${ }^{90} \mathrm{Sr} /{ }^{90} \mathrm{Y}$ nuclei were recorded by a scintillator equipped with a photomultiplier (Figure 1). Electronics made it possible to amplify and discriminate photomultiplier pulses before registering them.

The radiation-sensitive part of the apparatus was protected from direct penetration of neutrons and $\gamma$-quanta from reactor with the help of a small box made of lead bricks and borated polyethylene.

The measuring system was located at a distance of about 20 meters from the core of a pulsed reactor.

Preliminary measurements showed that in the absence of the $\beta$-source, the measuring system, when the reactor is operating, gives false positives no more than once every few minutes.

Additional measurements have shown that this phenomenon is absent when the reactor is switched off and the beta-source creates a statistically uniform count in all channels.

The measurement results are shown in Figure 3.

From these data it can be seen that, at the reactor flash, there is indeed some visible increase of beta-decays intensity.

\section{Conclusions}

Thus, the results of measurements show that the flux of reactor neutrinos does 
indeed have some quite noticeable effect on the phenomenon of beta-decay.

At that, the value of the ratio of flows is close to

$$
\frac{\Phi_{\text {react }}}{\Phi_{\text {cosm }}} \approx 3 \times 10^{-4} \text {. }
$$

This confirms the point of view on this problem that the phenomenon of beta-decay is a consequence of the neutrino flux action.

The discovered phenomenon of the impact of reactor neutrinos on beta-decay suggests that the modern approach to the problem of beta-decay should be revised. Many scientists dealing with this problem have argued that the theory of beta-decay in its present state is unsatisfactory [10]. Naturally, the fact that the beta-decay is a consequence of the impact of neutrino flux on nuclei requires the creation of a new theory of this phenomenon.

The author is sincerely grateful to Valery V. Zhuravlev for careful measurements at the reactor IBR-2 and to Evgeny P. Shabalin for discussing the problem of short-lived isotopes.

\section{Conflicts of Interest}

The author declares no conflicts of interest regarding the publication of this paper.

\section{References}

[1] Jenkins J.H., et al. (2009) Astroparticle Physics, 32, 42-46

[2] Falkenberg, E.D. (2001) Apeiron, 8, 32-45. https://pdfs.semanticscholar.org/3a21/346203f836847e60016770d931b324a46be9.pdf

[3] Vasiliev, B.V. (2017) Journal of Modern Physics, 8, 338-348. http://www.scirp.org/Journal/PaperInformation.aspx?PaperID=74443 https://doi.org/10.4236/jmp.2017.83023

[4] Vasiliev, B.V. (2015) Journal of Modern Physics, 6, 648-659. http://www.scirp.org/Journal/PaperInformation.aspx?PaperID=55921 https://doi.org/10.4236/jmp.2015.65071

[5] Serebrov, A.P. (2005) Physics-Uspekhi, 48, 867-885. http://ufn.ru/ru/articles/2005/9/a/ https://doi.org/10.1070/PU2005v048n09ABEH003536

[6] SNO Collaboration, Ahmed, S.N., Anthony, A.E., Beier, E.W., et al. (2004) arXiv:nuclyex 0309004

[7] Hayes, A.C. and Vogel, P. (2016) arXiv:1605.02047v1. https://arxiv.org/pdf/1605.02047.pdf

[8] Krane, K.S. (1988) Introductory Nuclear Physics. John Wiley and Sons, Hoboken.

[9] Kikoine, I.K. (1978) Physical Tables. Atomizdat, Moscow. (In Russian)

[10] Frauenfelder, H. and Henly, E. (1974) Subatomic Physics. Prentice-Hall, Englewood Cliffs, NJ, USA. 